\title{
Independent Modulation of von Willebrand Factor and Fibrinogen Binding to the Platelet Membrane Glycoprotein Ilb/lla Complex as Demonstrated by Monoclonal Antibody
}

\author{
Vincenzo Trapani Lombardo, Elisabeth Hodson, James R. Roberts, \\ Thomas J. Kunicki, Theodore S. Zimmerman, and Zaverio M. Ruggeri \\ Departments of Basic and Clinical Research and of Immunology, Scripps Clinic and Research Foundation, La Jolla, California 92037
}

\section{Abstract}

In this study we have used two new monoclonal antibodies, designated LJP5 and LJP9, as well as a previously described one, AP2, all specific for the platelet membrane glycoprotein (GP)IIb/ IIIa complex. None of them reacted with dissociated GPIIb or GPIIIa. The monovalent Fab fragment of both LJP5 and LJP9 bound to unstimulated platelets in a saturable manner, but binding was markedly decreased after platelets had been incubated at $37^{\circ} \mathrm{C}$ in the absence of added extracellular calcium. The binding of LJP9 was not affected by AP2, but was blocked by excess LJP5. On the contrary, the binding of LJP5 was blocked in the presence of both AP2 and LJP9. Thus, these antibodies bound to distinct epitopes of GPIIb/IIIa. At saturation, the binding to unstimulated platelets was between 2.41 and $10.9 \times 10^{4}$ molecules/platelet for LJP5 and between 3.47 and $9.1 \times 10^{4}$ molecules/platelet for LJP9 (range of 11 and 10 experiments, respectively). Binding increased up to $\mathbf{5 0 \%}$ after thrombin stimulation. The estimated association constant, $K_{n}$, was $2.7 \times 10^{7}$ $\mathrm{M}^{-1}$ for LJP5 and $3.85 \times 10^{7} \mathrm{M}^{-1}$ for LJP9. Both LJP5 and LJP9 partially inhibited the association of ${ }^{45} \mathrm{Ca}^{2+}$ with the surface of unstimulated platelets. Moreover, both antibodies blocked the binding of von Willebrand factor (vWF) to stimulated platelets, whereas only LJP9, but not LJP5, blocked fibrinogen binding. LJP9 was also a potent inhibitor of platelet aggregation, whereas LJP5 was without effect in this regard. The results of the present study demonstrate that independent modulation of vWF and fibrinogen binding to stimulated platelets can be attained with monoclonal antibodies directed against distinct epitopes of GPIIb/IIIa.

\section{Introduction}

The multimeric glycoprotein von Willebrand factor (vWF) ${ }^{1}$ plays an essential role in the formation of a normal platelet plug at

This work was presented in part at the 26th Meeting of the American Society of Hematology, 1-4 December 1984, Miami, FL and was published as an abstract (1984. Blood. 64[Suppl. 1]:249a).

Dr. Kunicki is with The Blood Center of Southeastern Wisconsin, Milwaukee. Address reprint requests to Dr. Ruggeri.

This is Publication No. 3629 BCR from the Research Institute of the Scripps Clinic.

Received for publication 31 December 1984 and in revised form 7 June 1985.

1. Abbreviations used in this paper: ACD, acid/citrate/dextrose; GP, platelet membrane glycoprotein; vWF, von Willebrand factor.

J. Clin. Invest.

(c) The American Society for Clinical Investigation, Inc. 0021-9738/85/11/1950/09\$1.00

Volume 76, November 1985, 1950-1958 sites of vascular injury, but the exact molecular mechanisms underlying this function have yet to be unraveled (1). Its specific binding to the platelet membrane glycoprotein (GP)Ib can be experimentally demonstrated using ristocetin $(2,3)$ or after desialylation of the molecule (4). More recently, it has been shown that thrombin, as well as other platelet agonists, also induce the binding of $\mathrm{vWF}$ to platelets $(5,6)$. In this case, however, binding occurs at site(s) localized on the GPIIb/IIIa complex $(2,7)$.

Fibrinogen is also essential for normal platelet aggregation and is thought to function by interacting with a specific receptor on GPIIb/IIIa $(8,9)$. Fibrinogen can inhibit vWF binding to stimulated platelets (10-13), and the reverse also applies (10). Moreover, synthetic peptides representing the carboxyl terminal of the $\gamma$ chain of fibrinogen, and much smaller than the parent molecule, can block the binding of both fibrinogen and vWF to GPIIb/IIIa $(10,14)$, thus suggesting that the site involved is very similar, if not identical, for both ligands. Similar conclusions have been reached based on experiments with monoclonal antibodies that inhibited the binding of both fibrinogen and vWF, as well as fibronectin and thrombospondin, to thrombin-stimulated platelets (15). Hence, the concept has been developed that a common binding site on GPIIb/IIIa may mediate the interaction of a family of adhesive glycoproteins with stimulated platelets.

In order to test the latter hypothesis, we have undertaken the characterization of a group of monoclonal antibodies directed against GPIIb/IIIa to see whether they all behaved similarly in inhibiting the binding of vWF and fibrinogen to platelets. For these studies, the smallest possible immunological probe was used, namely the monovalent Fab fragment derived from purified monoclonal IgG. We have obtained evidence that blocking distinct epitopes of the GPIIb/IIIa complex has distinct effects on the binding of vWF and fibrinogen to stimulated platelets.

\section{Methods}

Monoclonal antibody and Fab fragment preparation. Monoclonal antibodies against human platelet membrane glycoproteins were produced by immunizing BALB/c mice with intraperitoneal injections of washed platelet suspensions. These were prepared by gel filtration of plateletrich plasma through Sepharose CL 2B (Pharmacia Fine Chemicals, Piscataway, $\mathrm{NJ}$ ) as previously described (2), the only difference being that no albumin was used in the eluting buffer. The first injection consisted of $10^{8}$ platelets in Freund's complete adjuvant. Two boosters of $10^{8}$ and $1.5 \times 10^{8}$ platelets, respectively, in Freund's incomplete adjuvant were given at 1 -wk intervals. A final booster of $3 \times 10^{8}$ platelets without adjuvant was given $4 \mathrm{~d}$ before fusion. Mouse spleen cells were fused with mouse plasmacytoma cells at a ratio of $6: 1$. The two new antibodies described in this report, designated LJP5 and LJP9, were derived from separate fusions. LJP5 was obtained using P3X63-Ag 8.653 plasmacytoma cells, and LJP9 using NS-1 cells. Fusion and growth of hybridomas were performed using standard technology, as previously described (16). Positive hybridomas producing anti-platelet antibodies were selected by 
enzyme-linked immunoadsorbent assay (17). This assay detected mouse IgG reacting with washed platelets bound to plastic microtiter wells by means of Poly-L-lysine hydrobromide, $M_{\mathrm{r}}=150,000-300,000$ (Sigma Chemical Co., St. Louis, MO). Positive hybridomas were subcloned twice by limiting cell dilution (18). Monoclonal antibody was then produced in mouse ascites fluid as described (16). The reference anti-GPIIb/IIIa monoclonal antibody, AP2, was prepared as previously described (19).

Purified IgG was obtained from ascites fluid as follows. AP2 (IgG1) and LJP9 (IgG2b) were isolated by binding to Protein A-Sepharose (Sigma Chemical Co.) followed by elution at pH 6 and 4.5, respectively (20). LJP5 (IgG1) did not bind to Protein A-Sepharose with a sufficiently high affinity, and was purified on DEAE Affi-Gel Blue (Bio-Rad Laboratories, Richmond, CA) (21). Subclass specificity of the IgG molecules was defined by Outcherlony immunodiffusion (22) against the appropriate antisera. Fab fragments were prepared from the purified IgG. Note that the terminology Fab has been used, for the sake of simplicity, even when IgG (as for LJP5 and LJP9) was digested with pepsin, in which case the monovalent fragment derived is more appropriately called Fab'. Fab refers properly to monovalent fragment obtained by papain digestion (as for AP2). LJP5 was digested to F $\left(\mathrm{ab}^{\prime}\right)_{2}$ with $10 \%$ (wt/wt) pepsin (Sigma Chemical Co.) in a $0.116 \mathrm{M}$ acetate buffer, $\mathrm{pH} 3.8$, containing $0.05 \mathrm{M}$ $\mathrm{NaCl}$, for $5 \mathrm{~h}$ at $37^{\circ} \mathrm{C}(23,24)$. The optimum conditions for digestion $(\mathrm{pH}$, time of incubation, pepsin concentration) were determined experimentally in each case. After dialysis in $0.02 \mathrm{M}$ Tris, $0.15 \mathrm{M} \mathrm{NaCl}$ buffer, pH 7.4, reduction to Fab was achieved with $0.01 \mathrm{M}$ cysteine for $60 \mathrm{~min}$ at $37^{\circ} \mathrm{C}$, followed by S-carboxymethylation with $0.03 \mathrm{M}$ iodoacetamide for $60 \mathrm{~min}$ at room temperature $\left(22-25^{\circ} \mathrm{C}\right)$. LJP9 was digested similarly to LJP5, but the pH used was 4.5 to avoid excessive digestion of the IgG. Monovalent Fab could be isolated directly from the digestion mixture by high-pressure molecular sieve chromatography (Waters Associates, Milford, MA) using two columns (dimensions $60 \times 2.15 \mathrm{~cm}$, Bio-Rad) mounted in series, one packed with TSK gel G-4000 SW and the other with G-2500 SW (Bio-Rad). The other main product isolated from the digestion mixture was $\mathrm{Fab} / \mathrm{c}$, as well as undigested IgG, in accordance with the previously reported fragmentation pattern of mouse IgG2b (24). AP2 (IgG1) was too susceptible to pepsin digestion for Fab to be obtained in satisfactory quantities. Therefore, this IgG was digested with $5 \%$ papain (wt/wt) (Sigma) in $0.15 \mathrm{M}$ phosphate buffer, $\mathrm{pH} 7$, for $90 \mathrm{~min}$ at $37^{\circ} \mathrm{C}$ (25). The mixture contained also $0.01 \mathrm{M}$ cysteine and $0.002 \mathrm{M}$ EDTA. The reaction was terminated by addition of $0.05 \mathrm{M}$ iodoacetamide for $60 \mathrm{~min}$ at room temperature. This achieved, at the same time, S-carboxymethylation of Fab. Undigested Fc was separated from the mixture by passage through a Protein A-Sepharose column to which Fab did not bind. All Fab preparations were finally concentrated to between 1.5 and $4 \mathrm{mg} / \mathrm{ml}$ by ultrafiltration and extensively dialyzed against $0.02 \mathrm{M}$ Tris, $0.15 \mathrm{M} \mathrm{NaCl}$ buffer, $\mathrm{pH}$ 7.4. Polyacrylamide gel electrophoresis $(10 \%$ acrylamide with $5 \%$ cross-linking) in the presence of sodium dodecyl sulfate (SDS) (26) was used to assess the purity of each Fab preparation. Protein concentration was measured by the method of Lowry et al. (27), using bovine serum albumin (Sigma Chemical Co.) and bovine IgG (Miles Scientific, Naperville, IL) as standards, or by light absorption at $280 \mathrm{~mm}$ assuming $\mathrm{E}_{280}^{1 \%}=14.2$.

Preparation of washed platelet suspensions. Washed platelets for aggregation and binding studies were prepared from blood drawn through 19-gauge needles into polypropylene syringes and transferred into polypropylene tubes containing 1/6th final volume of acid/citrate/dextrose (ACD), $\mathrm{pH}$ 4.5. Platelet-rich plasma was obtained as previously described (2). Platelets were routinely washed free of plasma constituents by the albumin density-gradient technique of Walsh et al. (28), with minor modifications. These consisted in the addition of apyrase (grade III, Sigma) to the platelet-rich plasma ( $5 \mathrm{U} / \mathrm{ml}$ based on 5 -ATPase activity) and to the buffer used in the two subsequent washes $(1$ and $0.2 \mathrm{U} / \mathrm{ml}$, respectively). The platelets were finally resuspended in Tyrode's buffer, $\mathrm{pH}$ 7.4 , and counted before use (2). Platelet suspensions devoid of divalent cations were obtained as described by Marguerie et al. (29). The calcium content of the buffers used for the latter procedure was $<5 \mu \mathrm{M}$, as determined by absorption spectrophotometry.

Surface labeling of platelet membrane glycoproteins. Blood was drawn in ACD and platelet-rich plasma prepared as described (2). The platelets were then pelleted at $2,000 \mathrm{~g}$ for $8 \mathrm{~min}$ at room temperature, in polypropylene tubes, and subsequently resuspended in a buffer composed of $0.01 \mathrm{M}$ Tris, $0.002 \mathrm{M}$ EDTA, $0.15 \mathrm{M} \mathrm{NaCl}, \mathrm{pH} 7.4$ (Tris-EDTA). ACD at a final concentration of $15 \%(\mathrm{vol} / \mathrm{vol})$ was also added. The platelet suspension was pelleted once more, and the platelets were then resuspended in Tris-EDTA at a count of $1 \times 10^{9} / \mathrm{ml} .1 \mathrm{mCi}$ of carrier-free $\mathrm{Na}{ }^{125}$ I (Amersham Corp., Arlington Heights, IL) was added to each $\mathrm{ml}$ of the platelet suspension, followed by $10 \mu$ l of $2.5 \mathrm{nM}$ lactoperoxidase (Sigma Chemical Co.). The mixture was kept in agitation with gentle stirring. Five successive $10-\mu \mathrm{l}$ aliquots of $0.003 \mathrm{M} \mathrm{H}_{2} \mathrm{O}_{2}$, diluted in the same Tris-EDTA buffer, were added at 10-s intervals, and the reaction was then terminated by diluting the mixture with 9 vol of Tris-EDTA buffer. The labeled platelets were washed twice in Tris-EDTA containing $\mathrm{ACD}$, and finally resuspended in a buffer composed of $0.05 \mathrm{M}$ Tris, 0.15 $\mathrm{M} \mathrm{NaCl}$, pH 7.4, containing $200 \mu \mathrm{g} / \mathrm{ml}$ leupeptin (Chemicon Cal-Med, El Segundo, CA), $0.01 \mathrm{M}$ EDTA and $0.005 \mathrm{M} N$-ethylmaleimide (Sigma Chemical Co.). The platelet count was adjusted to $4 \times 10^{9} / \mathrm{ml}$, and lysis was achieved by addition of $1 \%$ (vol/vol) Triton X-100 (Eastman Kodak Co., Rochester, NY) in 30 min at $4^{\circ} \mathrm{C}$ with constant agitation. The mixture was then centrifuged at $100,000 \mathrm{~g}$ for $30 \mathrm{~min}$ at $4^{\circ} \mathrm{C}$, and the solubilized membrane glycoproteins remaining in the supernatant were aliquoted and stored at $-70^{\circ} \mathrm{C}$ until used.

Immunoisolation of platelet membrane glycoproteins. This was performed following a technique recently described (30). In brief, polyvinyl chloride microtiter plates (Dynatech Laboratories, Alexandria, VA) were coated with $10 \mu \mathrm{g}$ of affinity-purified rabbit anti-mouse IgG (Jackson ImmunoResearch Laboratories, Avondale, PA) dissolved in phosphate buffer, $\mathrm{pH} 7.2$, as indicated (30). After overnight incubation at $4^{\circ} \mathrm{C}$, the plates were washed and the monoclonal antibody to be tested (usually culture fluid supernatant or ascites) was added to several wells (usually four), followed by incubation for $4 \mathrm{~h}$ at room temperature. After washing. blocking of unreacted sites on the plastic, and additional washing, 100 $\mu \mathrm{l}$ of radiolabeled platelet membrane proteins, prepared as described above, was added to each well and incubated for $4 \mathrm{~h}$ at room temperature. The plates were then washed four times with phosphate buffer containing $0.5 \%$ Triton and $0.1 \%$ SDS, after which $50 \mu$ of hot (boiling) $0.01 \mathrm{M}$ phosphate buffer, $\mathrm{pH} 7.0$, containing $2 \%$ SDS, $5 \%$ 2-mercaptoethanol, $10 \%$ glycerol, and $0.05 \%$ bromophenol blue was added to each well. After incubation for $5 \mathrm{~min}$, the samples from wells containing the same monoclonal antibody were pooled, boiled for an additional $5 \mathrm{~min}$, and then analyzed in SDS-polyacrylamide gel electrophoresis.

Crossed immunoelectrophoresis of platelet membrane glycoproteins. This was performed as previously described (31), using $100 \mu \mathrm{g}$ of platelet lysate in the first dimension and running the second dimension against polyspecific rabbit anti-human platelet antibody (purified IgG). Purified $\mathrm{IgG}$ of the monoclonal antibody to be tested $(50 \mu \mathrm{g})$ was incorporated in the intermediate gel of the second dimension. After electrophoresis, gels were washed twice and then incubated with at least $4 \times 10^{6} \mathrm{cpm}$ $(\sim 80 \mu \mathrm{g})$ of ${ }^{125}$ I-labeled affinity-purified rabbit anti-mouse IgG (Zymed Laboratories, Inc., San Francisco, CA). Following an 18-h incubation at ambient temperature in a humidified chamber, the gels were washed, dried and stained with Coomassie blue-R (Sigma Chemical Co.) as previously described (31). Autoradiography was accomplished by storing dried gels in the dark for 4-24 h between a Kodak X-Omat R film and a duPont Cronex Lightning Plus screen (E. I. du Pont de Nemours, Inc., Wilmington, DE), at ambient temperature.

Aggregation studies. Platelet-rich plasma for aggregation studies was prepared as described above, the only difference being that blood was anticoagulated with $0.11 \mathrm{M}$ trisodium citrate in a proportion of 9:1. For these experiments, the platelet count in platelet-rich plasma or washed platelet suspensions was adjusted so that the final count in the experimental mixture was $2 \times 10^{8} / \mathrm{ml}$. The effect of monoclonal Fab on platelet aggregation was tested by incubating the $\mathrm{Fab}$ and the platelets for $3 \mathrm{~min}$ at $37^{\circ} \mathrm{C}$, without stirring, before transferring to the stirred cell of the aggregometer and adding the agonist. When washed platelets were used, purified fibrinogen $\left(300 \mu \mathrm{g} / \mathrm{ml}, 8.8 \times 10^{-7} \mathrm{M}\right)$ and $\mathrm{CaCl}_{2}(2 \mathrm{mM})$ were added to the mixture together with the antibody. Aggregation was re- 
corded as increase in light transmission through the mixture using a Lumiaggregometer (Chrono Log Corporation, Havertown, PA) and a dual-channel recorder (Kipp and Zonen, Bohemia, NY).

Purification of fibrinogen and $v W F$. Fibrinogen was purified from blood collected into $1 / 10$ volume of $0.11 \mathrm{M}$ trisodium citrate anticoagulant, containing $1 \mathrm{M} \epsilon$-aminocaproic acid and $0.365 \mathrm{mg} / \mathrm{ml}$ of Aprotonin (Sigma Chemical Co.), by means of precipitation with polyethylene glycol 1,000 (Sigma Chemical Co.) as previously described (32). Analysis of the purified fibrinogen by $7.5 \%$ polyacrylamide ( $5 \%$ cross-linking) slab gel electrophoresis in the presence of SDS and under reducing conditions showed the characteristic $A \alpha, B \beta$, and $\gamma$ chains (33). Absence of vWF and fibronectin contamination was further established by immunoblotting (34) with monospecific antibodies. The purified fibrinogen was at least $92 \%$ clottable.

vWF was purified following methods previously described in detail $(2,35,36)$. Analysis of the purified vWF by $5 \%$ polyacrylamide $(5 \%$ cross-linking) slab gel electrophoresis in the presence of SDS and under reducing conditions demonstrated one major band of apparent $M_{\mathrm{r}}$ $=220,000$. In particular, no fibrinogen was detected in the vWF preparations (2). Absence of fibrinogen and fibronectin contamination was further established by immunoblotting with monospecific antibodies. All preparations of purified $\mathrm{vWF}$ had $>100 \mathrm{U}$ of ristocetin cofactor activity and vWF antigen per milligram of protein, and had a multimeric structure like that of plasma vWF (2).

Radioiodination of proteins. Purified proteins were radiolabeled with carrier-free $\mathrm{Na}^{125}$ I (Amersham Corp.) using Iodogen (Pierce Chemical Co., Rockford, IL), as described by Fraker and Speck (37). All radiolabeled proteins were analyzed by polyacrylamide gel electrophoresis and showed no structural alterations as compared to the unlabeled counterparts. Specific activity was between 0.4 and $1.2 \mathrm{mCi} / \mathrm{mg}$ of protein for all ligands. Radioactivity was over $90 \%$ precipitable in $10 \%$ trichloroacetic acid in all cases.

Binding studies. The binding of radiolabeled anti-GPIIb/IIIa Fab to platelets was tested with both platelet-rich plasma and washed suspensions. Fab and platelets (final count between 0.3 and $1 \times 10^{8} / \mathrm{ml}$ ) were incubated for $30 \mathrm{~min}$ at $22-25^{\circ} \mathrm{C}$, and platelets were then separated from the reaction mixture by layering duplicate aliquots of the latter onto $20 \%$ sucrose in a microcentrifuge tube (2). After centrifugation at $13,000 \mathrm{~g}$ for $4 \mathrm{~min}$ (Beckman Instruments, Inc., Fullerton, CA), the tip of the tubes containing the pelleted platelets was cut with a scalpel and the platelet-bound radioactivity counted in a gamma-scintillation counter (Packard Instrument Co., Inc., Downers Grove, IL). Nonspecific binding was determined in the presence of at least a 20 -fold excess of the correspondent unlabeled Fab and never exceeded $10 \%$ of total binding. The number of antibody molecules bound per platelet was estimated by measuring binding at antibody concentrations where a twofold increase in concentration gave $<5 \%$ increase in binding. The association constant, $K_{\mathrm{a}}$, was then calculated as the reciprocal of the free antibody concentration that gave half maximal binding, as derived from the binding isotherm. These binding parameters were also obtained by the method of Scatchard (38), assuming one class of non-interacting binding sites, and the results were in close agreement.

The binding of radiolabeled vWF and fibrinogen to platelets was determined after stimulation with either $\alpha$-thrombin (the generous gift of Dr. J. W. Fenton II, New York State Department of Health, Albany, NY) or ADP (Sigma Chemical Co.). To measure thrombin-induced binding, platelets at a concentration of $5 \times 10^{8} / \mathrm{ml}$ were stimulated with $0.1 \mathrm{U} / \mathrm{ml}$ of thrombin for $10 \mathrm{~min}$ at $22-25^{\circ} \mathrm{C}$. Hirudin (Sigma Chemical Co.) was then added at a 16-fold excess (U/U). After $5 \mathrm{~min}$ at $22-25^{\circ} \mathrm{C}$, radiolabeled ligand was added at the desired concentrations and incubation continued for 30 min (platelet count at this point: $1 \times 10^{8} / \mathrm{ml}$ ). Platelets were then separated from the reaction mixture as described above. The binding induced by $\operatorname{ADP}(20 \mu \mathrm{M})$ was measured by incubating the latter with platelets, radiolabeled ligand, and $\mathrm{CaCl}_{2}(2 \mathrm{mM})$ all mixed at the same time. Nonspecific binding was always measured with a 20 100 -fold excess of the corresponding unlabeled ligand. To test the effect of the monoclonal Fab fragments on binding, the desired concentration of antibody was added to the platelet mixture immediately before the other reagents. Nonspecific binding was substracted from total binding to calculate specific binding. Nonspecific binding was never more than $25 \%$ of total binding, and was essentially the same whether LJP5 and LJP9 were present in the mixture or not. Binding isotherms were fitted to the experimental data, each one the mean of duplicate readings which agreed within $20 \%$, using least square regression analysis.

The binding of ${ }^{45} \mathrm{Ca}^{2+}$ (Amersham Corp.) to the surface of unstimulated washed platelets was measured as described by Brass and Shattil (39), using free $\mathrm{Ca}^{2+}$ concentrations, in EGTA buffer, between $10^{-6}$ and $10^{-8} \mathrm{M}$. For these studies, washed platelets devoid of calcium were prepared as described above (29), with the exception that the final suspension contained $1 \mathrm{mM} \mathrm{Mg}^{2+}$ and was incubated with $1 \mathrm{mM}$ acetylsalicilic acid for $30 \mathrm{~min}$ at room temperature before use.

\section{Results}

The two new anti-platelet monoclonal antibodies here described were designated LJP5 and LJP9. In crossed immunoelectrophoresis, they reacted against the membrane GPIIb/IIIa complex (Fig. 1), but not against dissociated GPIIb/IIIa or GPIIIa (Fig. 2). Furthermore, immunoisolation studies using surface-labeled platelets demonstrated that both LJP5 and LJP9 reacted with two proteins exhibiting apparent molecular weight correspondent to that of GPIIb and GPIIIa (Fig. 3). The same proteins reacted with AP2, a well-characterized anti-GPIIb/IIIa monoclonal antibody $(19,37)$.

Monovalent Fab fragment of both LJP5 and LJP9 bound to unstimulated human platelets in a saturable manner (Fig. 4). The mean number of LJP5 Fab molecules bound per platelet, measured in 11 separate experiments with platelets from different donors, was 5.06 (range 2.41-10.9) $\times 10^{4}$, with an estimated association constant, $K_{\mathrm{a}}$, of $2.7 \times 10^{7} \mathrm{M}^{-1}$. The corresponding values for LJP9 Fab (10 experiments) were 5.48 (range 3.47$9.1) \times 10^{4}$ molecules/platelet, with $K_{\mathrm{a}}=3.85 \times 10^{7} \mathrm{M}^{-1}$. Binding to thrombin-stimulated platelets $(0.5 \mathrm{NIH} \mathrm{U} / \mathrm{ml})$ was increased up to $50 \%$ without apparent change in affinity. The binding of LJP5 to platelets was markedly reduced in the presence of a 20fold excess of AP2, whereas the binding of LJP9 was unaffected by AP2 (Fig. 4). On the other hand, LJP9 inhibited the binding of LJP5 and vice versa, and results were compatible with a mechanism of competitive inhibition (Fig. 4). The binding of AP2, however, was blocked by LJP5 but not by LJP9 (not shown here).

Platelets washed in a calcium-free environment retained the ability to bind both LJP5 and LJP9 when tested promptly after isolation, but binding decreased markedly in a time-dependent manner when platelets were incubated at $37^{\circ} \mathrm{C}$ before adding the antibodies. The decrease in binding was largely, but usually not completely, prevented by the addition of $\mathrm{Ca}^{2+}$ to the platelets kept at $37^{\circ} \mathrm{C}$. Platelets kept at $22-25^{\circ} \mathrm{C}$ in the absence of $\mathrm{Ca}^{2+}$ showed some decrease in the ability to bind both antibodies, but much less so than platelets kept at $37^{\circ} \mathrm{C}$ (Table I). Both LJP5 and LJP9 bound to unstimulated or thrombin-stimulated platelets in the presence of EDTA, at concentration as high as 6.4 $\mathrm{mM}$, when platelets were kept at room temperature and the $\mathrm{pH}$ of the suspension was 7.4 (Table II). Under the same conditions, binding of fibrinogen and vWF to thrombin-stimulated platelets was completely blocked (Table II). Both antibodies, however, failed to bind to platelets in the presence of EDTA when the mixtures were incubated at $37^{\circ} \mathrm{C}$ and the $\mathrm{pH}$ was raised to 8 . The association of ${ }^{45} \mathrm{Ca}^{2+}$ with the surface of unstimulated platelets was partially decreased in the presence of both LJP5 and LJP9 (Table III). 

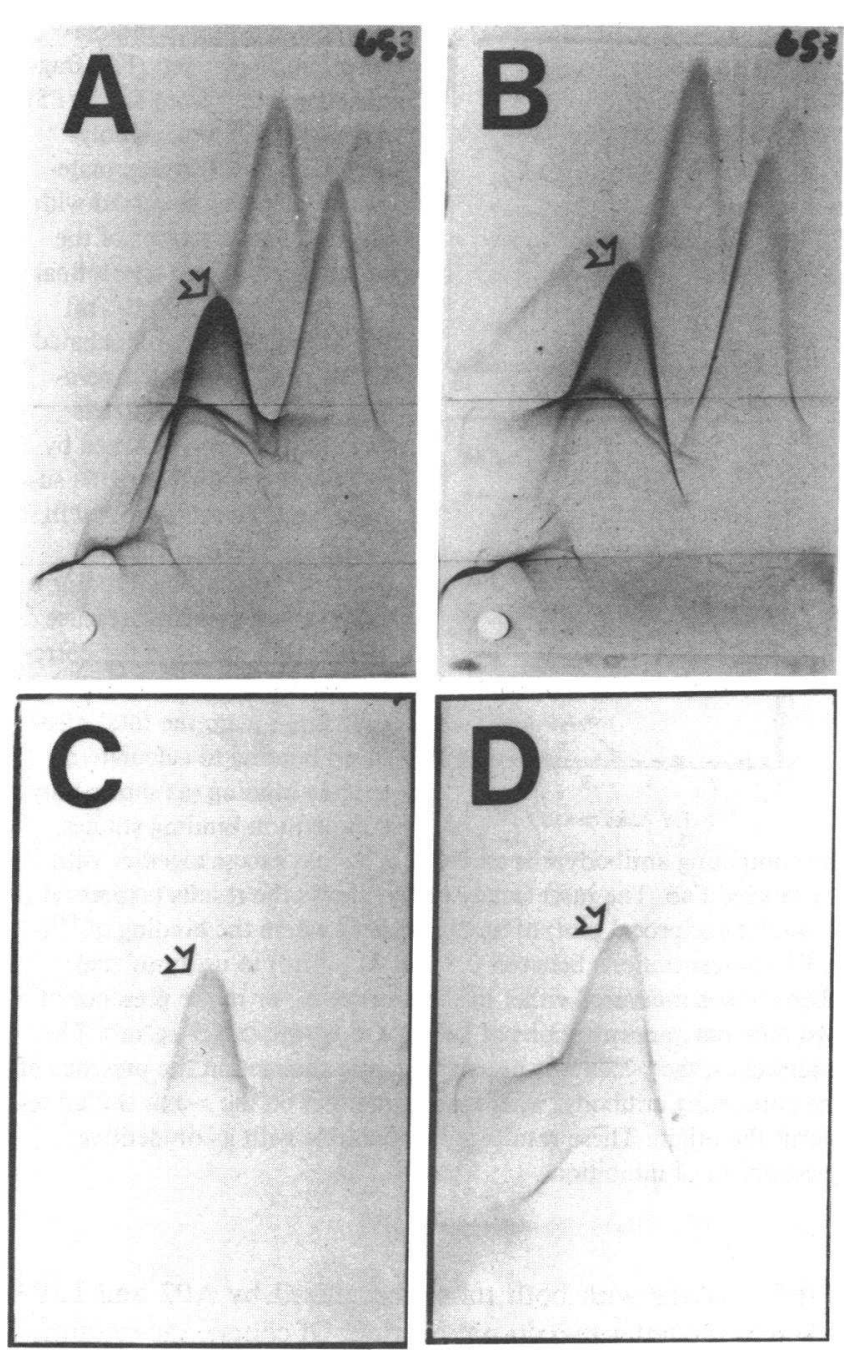

Figure 1. Specificity of LJP5 and LJP9 as determined by crossed immunoelectrophoresis. $100 \mu \mathrm{g}$ of protein, derived from washed platelets lysed in the absence of EDTA, was electrophoresed in the first dimension and then, at $90^{\circ}$, into a second-dimension gel containing: in the intermediate gel, $50 \mu$ l of hybridoma culture fluid from LJP9 (gel $A$ ) or LJP5 (gel $B$ ); in the upper gel, a polyspecific rabbit anti-human platelet antibody $\left(250 \mu \mathrm{g}\right.$ of purified IgG per $\left.\mathrm{cm}^{2}\right)$. After completion of electrophoresis, bound monoclonal antibody was detected by incubation of gels in buffer containing ${ }^{125}$ I-labeled affinity-purified rabbit anti-mouse IgG, followed by several washes to remove unbound radiolabeled antibody, and autoradiography. Coomassie blue-stained gels are depicted in $A$ and $B$. The corresponding autoradiographs are shown in $C$ and $D$, respectively. The precipitin arc given by the GPIIb/IIIa complex is indicated by an open arrow. Both LPP5 and LJP9 reacted with this complex.

The binding of vWF to ADP-stimulated platelets was completely blocked by LJP5 as well as LJP9 (Fig. 5, upper panel). The latter antibody also blocked the binding of fibrinogen, whereas LJP5 had no effect in this regard (Fig. 5, lower panel). Similar results were obtained with thrombin-stimulated platelets. The amount of each antibody necessary to achieve maximal inhibitory effect was in agreement with the amount that gave saturation of binding to platelets (compare Fig. 4 and Fig. 5). Nevertheless, LJP5 Fab had no effect on fibrinogen binding even when added in a 10-fold excess over the saturating amounts.
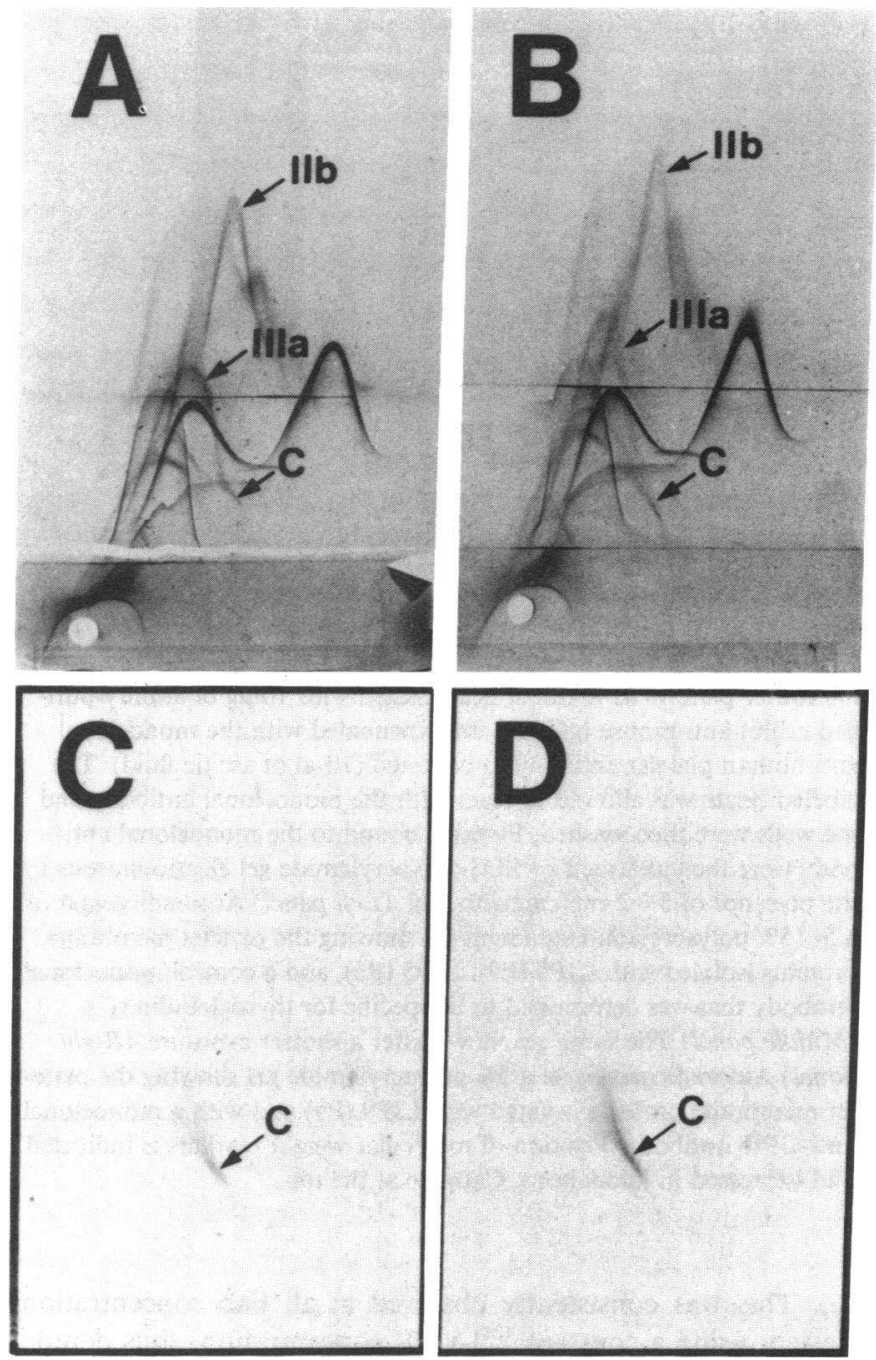

Figure 2. Specificity of LJP5 and LJP9 as determined by crossed immunoelectrophoresis. Legend as in Fig. 1, except that protein derived from platelets lysed in the presence of EDTA was analyzed. The precipitin arc containing the GPIIb/IIIa complex $(C)$ is markedly diminished, although still detectable. Additional precipitin arcs containing dissociated GPIIb and GPIIIa are indicated. Note that both LJP9 (gels $A$ and $C$ ) and LJP5 (gels $B$ and $D$ ) react with the residual GPIIb/IIIa complex, but neither with dissociated GPIIb nor GPIIla.

The concentration of $\mathrm{vWF}$ or fibrinogen added to the platelets had no effect on the extent of inhibition obtained with the antibodies, and results were compatible with a noncompetitive inhibitory mechanism (Fig. 5). The binding of vWF added at concentrations between 0.5 and $30 \mu \mathrm{g} / \mathrm{ml}$ could be completely inhibited by LJP5 and LJP9. LJP9 also markedly inhibited the binding of fibrinogen when the latter was added at concentrations between 5 and $600 \mu \mathrm{g} / \mathrm{ml}$, whereas LJP5 was consistently without effect at all concentrations of fibrinogen tested. Experiments on the inhibition of vWF and fibrinogen binding were performed several times (only three are reported in Fig. 5) using platelets from different donors and different Fab preparations. In all cases, the results were in close agreement. Neither LJP5 nor LJP9 affected vWF binding to platelets in the presence of ristocetin.

The inhibitory effect of LJP5 Fab on vWF binding to platelets was inversely related to the platelet count in the mixture (Fig. 


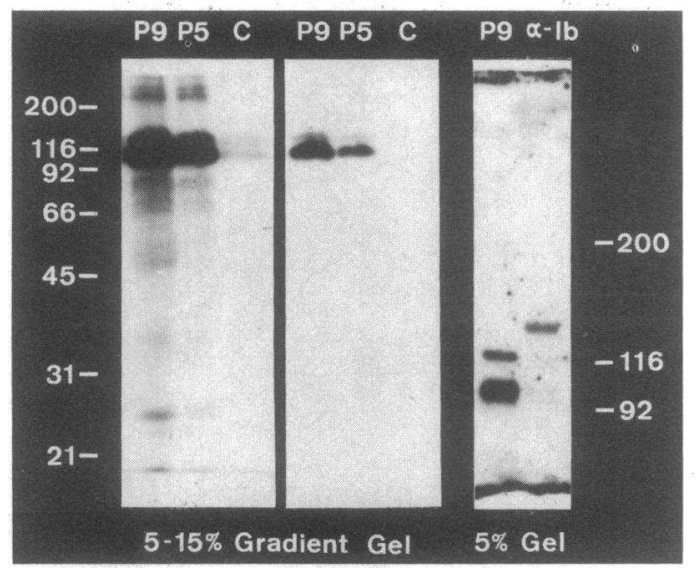

Figure 3. Specificity of LJP5 and LJP9 as determined by immunoisolation. Washed platelets $\left(4 \times 10^{9} / \mathrm{ml}\right)$ were surface labeled with ${ }^{125} \mathrm{I}$ and lysed with Triton X-100. $100 \mu \mathrm{l}$ of the labeled lysate was added to microtiter plate wells that had been coated with $10 \mu \mathrm{g}$ of affinity-purified rabbit anti-mouse IgG and then incubated with the monoclonal anti-human platelet antibody to be tested ( $10 \mu \mathrm{l}$ of ascitic fluid). The labeled lysate was allowed to react with the monoclonal antibody and the wells were then washed. Proteins bound to the monoclonal antibody were then analyzed by SDS-polyacrylamide gel electrophoresis in the presence of 5\% 2-mercaptoethanol. (Left panel) Autoradiograph of a 5-15\% polyacrylamide gradient gel showing the platelet membrane proteins isolated with LJP9 (P9), LJP5 (P5), and a control monoclonal antibody that was determined to be specific for thyroglobulin $(C)$. (Middle panel) The same gel shown after a shorter exposure. (Right panel) Autoradiography of a 5\% polyacrylamide gel showing the platelet membrane proteins isolated with LJP9 (P9) and with a monoclonal anti-GPIb antibody. Position of molecular weight markers is indicated and expressed in kilodaltons. Cathode at the top.

6). This was consistently observed at all Fab concentrations tested, using a constant ${ }^{125} \mathrm{I}-\mathrm{vWF}$ concentration, thus demonstrating that the effect of LJP5 Fab on vWF binding was not related to alterations induced in the vWF molecule, but rather to occupancy of the corresponding epitope on GPIIb/IIIa.

As shown in Fig. 4, excess LJP5 could block LJP9 binding to platelets. Accordingly, excess LJP5 Fab could effectively prevent the inhibitory effect of LJP9 Fab on fibrinogen binding (Fig. 7). Moreover, LJP5 Fab had no effect on ADP-induced platelet aggregation, whereas LJP9 Fab completely inhibited it (Fig. 8). Identical results were observed when $\alpha$-thrombin or collagen were used as agonists instead of ADP. In accordance with the results of fibrinogen binding studies, excess LJP5 Fab could prevent the inhibitory effect of LJP9 on aggregation (Fig. 8). The results of aggregation studies were identical for either citrated platelet-rich plasma or washed platelet suspensions containing purified fibrinogen and $2 \mathrm{mM} \mathrm{CaCl}$.

\section{Discussion}

Our results demonstrate that the two new monoclonal antiplatelet antibodies described in this report, LJP5 and LJP9, recognize distinct epitopes on the GPIIb/IIIa complex that are also different from the one recognized by AP2. In fact, LJP9 and AP2 bound to platelets independently of each other, whereas LJP5 and AP2 competed for binding. Competition for binding was also observed between LJP5 and LJP9. The most likely explanation for our findings is that the epitope recognized by

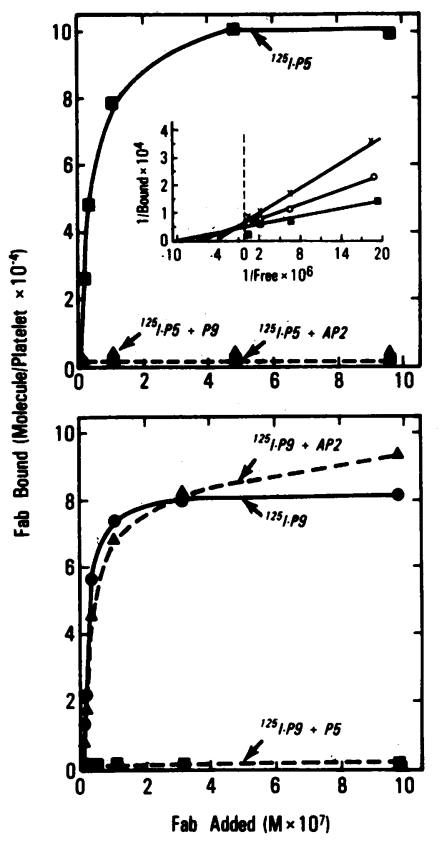

Figure 4. Binding of radiolabeled Fab to platelets. Fab fragments prepared from LJP5 (P5) and LJP9 (P9) were radiolabeled with ${ }^{125} \mathrm{I}$. Citrated platelet-rich plasma was mixed with varying concentrations of the radiolabeled Fab to give a final platelet count of $3 \times 10^{7} / \mathrm{ml}$ and the mixture was incubated for $30 \mathrm{~min}$ at room temperature. Separation of platelets from free Fab was attained by centrifugation through $20 \%$ sucrose, and the radioactivity in the platelet pellets was then counted. Nonspecific binding was measured in the presence of a 20-fold excess of the correspondent unlabeled Fab, and subtracted from the total measured binding to calculate the specific binding (as shown). In competition binding studies,

the competing antibody was added at a 20-fold excess together with the labeled Fab. The inset (upper panel) shows the results (expressed as double reciprocal plot) of an experiment where the binding of ${ }^{125} \mathrm{I}$ LJP5 (concentrations between 0.5 and $31 \mu \mathrm{g} / \mathrm{ml}$ ) to unstimulated platelets was measured either in the absence $(a)$ or in the presence of two different concentrations of LJP9 $(x, 6 \mu \mathrm{g} / \mathrm{ml} ; \circ, 1.5 \mu \mathrm{g} / \mathrm{ml})$. The intersect on the $y$-axis was not significantly changed in the presence of the competing antibody, whereas the intersect on the $x$-axis shifted towards the origin. These results are compatible with a competitive mechanism of inhibition.

LJP5 overlaps with both those recognized by AP2 and LJP9, whereas the latter two do not overlap. Of course, the resolution of these inhibition binding studies is limited by the size of the

Table I. Effect of Temperature on LJP5 and LJP9 Binding to Platelets in the Absence of Added Extracellular Calcium

\begin{tabular}{lllr}
\hline & & \multicolumn{2}{l}{ Antibody bound } \\
\cline { 3 - 4 } Temperature & Incubation time & LJP5 & LPP9 \\
\hline${ }^{\circ} \mathrm{C}$ & $\min$ & $\%$ & \\
37 & 0 & 100 & 100 \\
& 15 & 38 & 34 \\
& 30 & 22 & 22 \\
& 45 & 13 & 12 \\
& 60 & 4 & 7 \\
$22-25$ & $60\left(\mathrm{Ca}^{2+}\right)$ & 68 & 73 \\
& 60 & 85 & 69
\end{tabular}

Platelets were incubated in the absence of added extracellular calcium (unless indicated) for variable periods of time before addition of the labeled antibodies. After this, incubation was continued at room temperature $\left(22-25^{\circ} \mathrm{C}\right)$ for additional $30 \mathrm{~min}$. When indicated, calcium was added at $2 \mathrm{mM}$ from the beginning of the incubation. Both antibodies were added at a concentration of $6 \mu \mathrm{g} / \mathrm{ml}\left(1.2 \times 10^{-7} \mathrm{M}\right)$ and the binding measured corresponded to that shown in Fig. 4 . The platelet count was $1 \times 10^{8} / \mathrm{ml}$ in the incubation mixtures, and the $\mathrm{pH}$ was 7.4. 
Table II. Effect of EDTA on the Binding of LJPS, LJP9, vWF and Fibrinogen to Thrombin-stimulated Platelets Kept at Room Temperature and pH 7.4

\begin{tabular}{llllrr}
\hline & & \multicolumn{3}{l}{ Binding } & \\
\cline { 3 - 6 } EDTA & $\begin{array}{l}\text { Thrombin } \\
\text { stimulation }\end{array}$ & LJP5 & LJP9 & vWF & Fibrinogen \\
\hline$m M$ & 0.15 NIH U/ml & $\%$ & $\%$ & $\%$ & $\%$ \\
0 & no & 100 & 100 & 0 & 0 \\
0 & yes & 148 & 115 & 100 & 100 \\
0.1 & yes & 129 & 107 & 91 & 89 \\
0.4 & yes & 147 & 99 & 0 & 0 \\
1.6 & yes & 121 & 125 & 0 & 0 \\
6.4 & yes & 107 & 122 & 0 & 0 \\
\hline
\end{tabular}

Platelets were stimulated with thrombin in the presence of EDTA for $10 \mathrm{~min}$, then hirudin was added followed by the radiolabeled ligands (see Methods). All incubations were at room temperature $\left(22-25^{\circ} \mathrm{C}\right)$ and the $\mathrm{pH}$ of the mixtures was 7.4. LJP5 and LJP9 were added at a concentration of $6 \mu \mathrm{g} / \mathrm{ml}\left(1.2 \times 10^{-7} \mathrm{M}\right)$, fibrinogen was at $60 \mu \mathrm{g} / \mathrm{ml}$, and $\mathrm{vWF}$ at $5 \mu \mathrm{g} / \mathrm{ml}$. Binding defined as $100 \%$ corresponded to that shown in Fig. 4 for the two antibodies, to 64,000 molecules/platelet for fibrinogen, and to $1.2 \mu \mathrm{g} / 10^{8}$ platelets for vWF.

Fab fragments used. Theoretically, conformational changes of GPIIb/IIIa following the binding of monoclonal antibody might also be responsible for the competition observed between LJP5 and the other two antibodies, even if the corresponding epitopes were not close to one another. The observed mechanism of competitive inhibition between LJP5 and LJP9, however, is not in favor of the latter hypothesis.

The average number of LJP5 and LJP9 molecules bound per platelet was $\sim 50,000$, a number not dissimilar from the ones previously reported for other anti-GPIIb/IIIa monoclonal antibodies (40-44). The range previously observed varied from $21,380(43)$ to $57,400(40)$ molecules/platelet, with one investigator reporting extreme values of $12,000-83,000(42)$. We also observed a wide range of values among different individual

Table III. Effect of LJP5 and LJP9 on the Association of ${ }^{45} \mathrm{Ca}^{2+}$ with the Surface of Unstimulated Platelets

\begin{tabular}{ll}
\hline Antibody present & ${ }^{45} \mathrm{Ca}^{2+}$ bound \\
\hline & sites/platelet \\
None & 24,900 \\
LP5 & 12,200 \\
LJP9 & 13,050 \\
\hline
\end{tabular}

Platelets were washed free of $\mathrm{Ca}^{2+}$ and with $1 \mathrm{mM} \mathrm{Mg}{ }^{2+}$ present (see Methods). Free ${ }^{45} \mathrm{Ca}^{2+}$ was then added at a concentration of 7.8 $\times 10^{-7} \mathrm{M}$, in $0.5 \mathrm{mM}$ EGTA buffer, and incubated with platelets (2 $\times 10^{8} / \mathrm{ml}$ ) for $10 \mathrm{~min}$ at $22-25^{\circ} \mathrm{C}$, either in the presence or absence of LJP5 and LJP9 $(30 \mu \mathrm{g} / \mathrm{ml})$. Separation of bound from free ${ }^{45} \mathrm{Ca}^{2+}$ was achieved by centrifugation through $20 \%$ sucrose. The amount of ${ }^{45} \mathrm{Ca}^{2+}$ bound to the platelet membrane was obtained by subtracting from the total value associated with the platelet pellet the amount that could not be displaced by the addition of $3 \mathrm{mM}$ EGTA 2 min before separation of bound from free ligand. The latter was considered to represent intracellular ${ }^{45} \mathrm{Ca}^{2+}$ that could not readily exchange with the extracellular medium (see Reference 39 ).

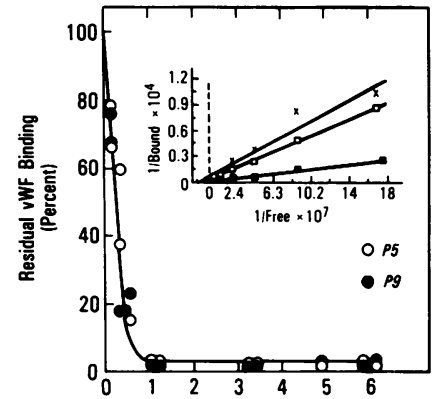

Figure 5. Effect of LJP5 and LJP9 on fibrinogen and vWF binding to platelets. Washed platelet suspensions $\left(1 \times 10^{8} \%\right.$ $\mathrm{ml}$ ) were mixed with $100 \mu \mathrm{g} / \mathrm{ml}$ of ${ }^{125}$ I-fibrinogen $\left(2.9 \times 10^{-7}\right.$ M) (lower panel), or $5 \mu \mathrm{g} / \mathrm{ml}$ of ${ }^{125} \mathrm{I}-\mathrm{vWF}$ (upper panel). This was followed by $0.002 \mathrm{M}$ $\mathrm{CaCl}_{2}$, the indicated concentration of Fab from LJP5 (P5) or LJP9 (P9), or an equal volume

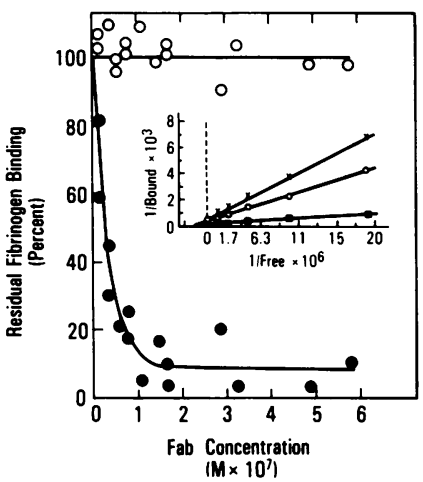
of Tyrode buffer, and $20 \mu \mathrm{M}$ ADP. All indicated concentrations were final. The mixture, containing also $2 \%$ bovine serum albumin, was incubated for $30 \mathrm{~min}$ at room temperature, after which the platelets were separated from free ligand by centrifugation through $20 \%$ sucrose. The radioactivity in the platelet pellets was then counted. Nonspecific binding was measured in the presence of a 100-fold excess of unla-

beled ligand. Specific binding was calculated by subtracting nonspecific from total binding, and expressed as percentage of the binding measured in the absence of Fab (as shown). The latter values corresponded to 38,600 molecules/platelet for fibrinogen and $0.6 \mu \mathrm{g} / 10^{8}$ platelets for vWF. These results are the aggregate of three separate experiments performed with different platelet preparations. The inset in the upper panel shows the results of a competition binding study where increasing amounts of ${ }^{125} \mathrm{I}-\mathrm{vWF}$ (between 1.3 and $37 \mu \mathrm{g} / \mathrm{ml}$ ) were added to platelets either in the absence $(\square)$ or in the presence of LJP5 $(\times, 1.5 \mu \mathrm{g} / \mathrm{ml} ; \square, 0.8 \mu \mathrm{g} / \mathrm{ml})$. The reciprocal of specific binding was plotted against the reciprocal of the free vWF concentration added. Even at the highest concentrations of vWF tested, the inhibitory effect of LJP5 could not be overcome. A change in position of the intersect on the $y$-axis, but not on the $x$-axis, was observed in the presence of the antibody, a finding compatible with a noncompetitive mechanism of inhibition. The inset in the lower panel shows the results of a similar experiment performed with ${ }^{125}$ I-fibrinogen (concentrations between 15 and $366 \mu \mathrm{g} / \mathrm{ml}$ ) either in the absence $(\square)$ or in the presence of LJP9 $(x, 15 \mu \mathrm{g} / \mathrm{ml} ; 0,3 \mu \mathrm{g} / \mathrm{ml})$. Results could be interpreted in a similar way as those obtained in the experiment with vWF. In both instances, platelets were stimulated with $0.1 \mathrm{NIH} \mathrm{U} / \mathrm{ml}$ of $\alpha$ thrombin (see Methods).

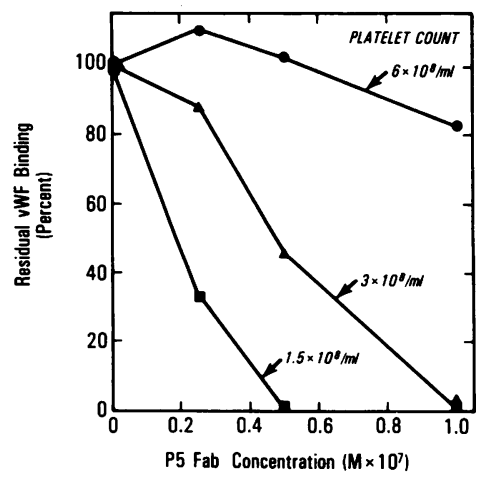

Figure 6. Effect of platelet count on inhibition of vWF binding by LJP5. The binding of ${ }^{125} \mathrm{I}-\mathrm{vWF}$ to platelets was measured as indicated in the legend to Figure 5, with the following modifications. The platelet count in the mixture varied between 1.5 and $6 \times 10^{8} / \mathrm{ml}$ (as indicated); and the platelets were stimulated with 0.1 NIH U/ml of $\alpha$-thrombin (see Methods). Binding of ${ }^{125} \mathrm{I}-\mathrm{vWF}$ in the presence of increasing concentrations of LJP5 Fab was expressed as percentage of the binding measured in the absence of LJP5 Fab. The latter value corresponded to $0.52 \mu \mathrm{g} / 10^{8}$ platelets. 


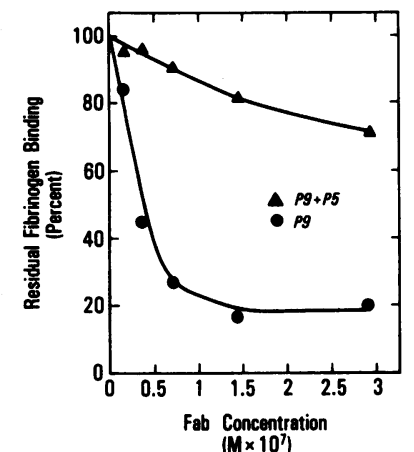

Figure 7. Effect of LJP5 on inhibition of fibrinogen binding by LJP9. The binding of ${ }^{125} \mathrm{I}$-fibrinogen to platelets was measured as indicated in the legend to Fig. 5, with the following modifications. In one series of mixtures, LJP9 Fab (P9) was present at the concentrations indicated on the abscissa. In a parallel series, LJP9 Fab was present at the same concentrations, but LJP5 Fab (P5) was also added at a 20 -fold excess. The results of ${ }^{125} \mathrm{I}$ fibrinogen binding were expressed as percentage of the binding measured in the absence of Fab.

platelets. These results may reflect technical variability of the assays, but are also likely to reflect individual variations in the number of GPIIb/IIIa molecules expressed on the platelet membrane. In our studies, we used monovalent Fab rather than divalent $\operatorname{IgG}(40,41,43,44)$ or $\mathrm{F}\left(\mathrm{ab}^{\prime}\right)_{2}(42)$. This may be responsible, at least in part, for some of the differences observed, since antibodies may bind to cell surface antigens divalently, and the proportion of divalent to monovalent binding may vary (45, 46). The reason why thrombin-stimulated platelets bound up to 50\% more LJP5 and LJP9 Fab than unstimulated platelets may reflect the fact that a pool of GPIIb/IIIa molecules contained in the $\alpha$-granule membrane becomes exposed on the platelet surface following secretion (47), thereby increasing the number of epitopes interacting with the monoclonal antibodies.

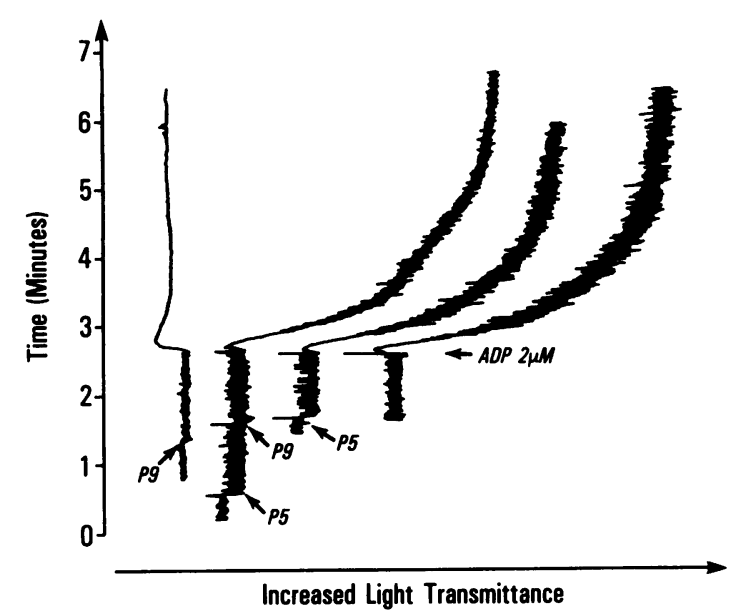

Figure 8. Effect of LJP5 and LJP9 on platelet aggregation. Washed platelet suspensions $\left(2 \times 10^{8} / \mathrm{ml}\right)$ were added to an aggregometer cuvette, under stirring conditions, and mixed with $0.002 \mathrm{M} \mathrm{CaCl}_{2}$ and $300 \mu \mathrm{g} / \mathrm{ml}$ of fibrinogen $\left(8.8 \times 10^{-7} \mathrm{M}\right)$. To the mixture was then added either LJP5 (P5) or LJP9 (P9) Fab separately ( $20 \mu \mathrm{g} / \mathrm{ml} \mathrm{each,}$ $\left.\sim 4.2 \times 10^{-7} \mathrm{M}\right)$, or LJP5 $(200 \mu \mathrm{g} / \mathrm{ml})$ followed by LJP9 $(20 \mu \mathrm{g} / \mathrm{ml})$. A control mixture contained Tyrode's buffer instead of Fab (curve on the right). All indicated concentrations were final. After equilibration and establishment of a straight baseline, ADP $(2 \mu \mathrm{M}$, final concentration) was added to the mixtures (at arrow) and platelet aggregation recorded as increased light transmittance through the cuvette. The decrease in light transmittance immediately after addition of ADP represents the platelet shape change. Note the inhibitory effect of LJP9 on platelet aggregation, but its lack of inhibition of the platelet shape change. Note also the lack of inhibition in the presence of LJP5 and the blocking effect of the latter on the inhibition exerted by LJP9.
Our results demonstrate that LJP5 and LJP9 recognize distinct epitopes on the platelet membrane whose appropriate conformation for binding the antibodies is calcium dependent. Similar results have been recently obtained with another antiGPIIb/IIIa monoclonal antibody (48). These findings do not prove that the epitopes recognized by LJP5 and LJP9 are formed by the association of GPIIb and GPIIIa, in that they might exist on either one of the two individual glycoproteins but only when the two are in complex. Nevertheless, the two epitopes can certainly be considered as complex dependent, inasmuch as they exist only under the conditions required for maintaining integrity of the GPIIb/IIIa complex (31, 49-52). Moreover, it is clear that the interaction of both LJP5 and LJP9 with the corresponding epitopes, while requiring the presence of the calcium-dependent heterodimer formed by GPIIb and GPIIIa, is independent of additional $\mathrm{Ca}^{2+}$ in the medium.

The two new antibodies here described, LJP5 and LJP9, showed marked differences in their effect on platelet function. The latter was a potent inhibitor of aggregation induced by ADP, collagen, or $\alpha$-thrombin, whereas the former was without effect in this regard. Accordingly, LJP9 blocked fibrinogen binding to stimulated platelets, but LJP5 did not. Both antibodies, however, completely blocked vWF binding to platelets stimulated by either ADP or thrombin. Recent studies have suggested that fibrinogen and vWF share a common binding site on GPIIb/IIIa (10, 14, 15). The most compelling evidence for this is provided by experiments performed with peptides representing the carboxyl terminal of the human fibrinogen $\gamma$ chain, which inhibit in a parallel fashion the binding of both fibrinogen and vWF to stimulated platelets $(10,14)$. Similar results have recently been obtained using monoclonal antibodies directed against GPIIb/IIIa (15). Our finding that LJP5 has contrasting effects on vWF and fibrinogen binding suggests, however, that the mechanisms involved in the interaction of these two ligands with platelets are, at least in part, distinct. This does not necessarily exclude the existence of a common receptor. Moreover, it is clear that the effects exerted by LJP5 and LJP9 are related to occupancy of the corresponding epitopes, in that LJP5 did not alter the binding properties of VWF, and displacement of LJP9 by LJP5 resulted in normal fibrinogen binding and platelet aggregation in the presence of both antibodies.

At present, it is not possible to explain in a definitive manner the mechanisms by which LJP5 and LJP9 have a differential effect on fibrinogen and vWF binding to stimulated platelets. In some aspects, the results of the present study are compatible with the concept that neither antibody directly blocks the specific binding site(s) for the two adhesive glycoproteins, because they both act as noncompetitive inhibitors of fibrinogen and/or vWF interaction with platelets. This conclusion must be taken with caution, however, as it relates to complex macromolecules whose binding to platelets is not fully reversible $(2,29,40)$. Two additional observations seem to support the concept that the epitopes recognized by LJP5 and LJP9 cannot be identified with the vWF and fibrinogen binding site(s). In fact, LJP5 and LJP9, but not fibrinogen nor vWF, bind to unstimulated platelets. Moreover, additional extracellular calcium, besides that required for formation of the GPIIb/IIIa complex, is not necessary for binding of the two antibodies to their respective epitopes, but it is for binding of vWF and fibrinogen to their receptor(s). These findings might be interpreted as evidence that the epitopes recognized by LJP5 and LJP9 are expressed independently of the vWF and fibrinogen binding site(s). However, they might also 
reflect the fact that calcium-dependent and stimulation-dependent changes in the microenvironment surrounding the GPIIb/ IIla complex are necessary for the binding of $\mathrm{vWF}$ and fibrinogen, but not of LJP5 and LJP9, possibly because of the larger size of the two adhesive glycoproteins as compared to the antibody molecules (53). The observations that smaller peptides derived from the fibrinogen $\gamma$ chain bind to unstimulated platelets (54), and that fibrinogen itself forms a complex with isolated GPIIb/ IIIa (8), are both in agreement with the latter hypothesis. Therefore, because vWF is obviously a much larger molecule than fibrinogen, it is possible that the steric constraints imposed by LJP5 on the microenvironment surrounding GPIIb/IIIa are less tight than those imposed by LJP9, so that only binding of the large vWF, but not fibrinogen, is affected by the former antibody. Both LJP5 and LJP9 interfered with the association of calcium with the membrane of unstimulated platelets, but only partially and to about the same extent. Therefore, changes in the $\mathrm{Ca}^{2+}$ associated with the GPIIb/IIIa complex are not likely to explain the different effect of the two antibodies on vWF and fibrinogen binding.

In conclusion, the present study demonstrates that the binding of at least two different adhesive glycoproteins to stimulated platelets can be independently modulated. This concept should prove important for unraveling the molecular bases of platelet interaction with specific ligands and for better understanding the mechanisms of platelet function.

\section{Acknowledgments}

We wish to thank Drs. Ed Plow and Mark Ginsberg for the stimulating discussion of these findings, Ms. Susan Russell for skilled technical assistance, Mrs. Linda Holland for performing preparative high-pressure liquid chromatography of Fab fragments, and Ms. Claire Jackson and Mrs. Ruth Stewart for excellent secretarial assistance.

This work was supported in part by grants HL-31950 and HL-15491 from the National Institutes of Health and grant RR-00833 from the U. S. Public Health Service.

\section{References}

1. Zimmerman, T. S., Z. M. Ruggeri, and C. A. Fulcher. 1983. Factor VIII/von Willebrand factor. Prog. Hematol. 13:279-309.

2. Ruggeri, Z. M., L. De Marco, L. Gatti, R. Bader, and R. R. Montgomery. 1983. Platelets have more than one binding site for von Willebrand Factor. J. Clin. Invest. 72:1-12.

3. Coller, B. S., E. I. Peerschke, L. E. Scudder, and C. A. Sullivan. 1983. Studies with a murine monoclonal antibody that abolishes ristocetin-induced binding of von Willebrand factor to platelets: Additional evidence in support of GPIb as a platelet receptor for von Willebrand factor. Blood. 61:99-110.

4. DeMarco, L., and S. S. Shapiro. 1981. Properties of human asialoFactor VIII. A ristocetin-independent platelet-aggregating agent. J. Clin. Invest. 168:321-328.

5. Fujimoto, T., S. Ohara, and J. Hawiger. 1982. Thrombin-induced exposure and prostacyclin inhibition of the receptor for factor VIII/von Willebrand factor on human platelets. J. Clin. Invest. 69:1212-1222.

6. Fujimoto, T., and J. Hawiger. 1982. Adenosine diphosphate induces binding of von Willebrand factor to human platelets. Nature (Lond.). 297:154-157.

7. Ruggeri, Z. M., R. Bader, and L. De Marco. 1982. Glanzmann thrombasthenia: deficient binding of von Willebrand factor to thrombinstimulated platelets. Proc. Natl. Acad. Sci. USA. 79:6038-6041.

8. Nachman, R. L., and L. L. K. Leung. 1982. Complex formation of platelet membrane glycoproteins IIb and IIIa with fibrinogen. J. Clin. Invest. 69:263-269.
9. Bennett, J. S., G. Vilaire, and D. B. Cines. 1982. Identification of the fibrinogen receptor on human platelets by photoaffinity labeling. $J$. Biol. Chem. 257:8049-8054.

10. Timmons, S., M. Kloczewiak, and J. Hawiger. 1984. ADP-dependent common receptor mechanism for binding of von Willebrand factor and fibrinogen to human platelets. Proc. Natl. Acad. Sci. USA. 81:4935-4939.

11. Schullek, J., J. Jordan, and R. R. Montgomery. 1984. Interaction of von Willebrand Factor with human platelets in the plasma milieu. $J$. Clin. Invest. 73:421-428.

12. Pietu, G., G. Cherel, G. Marguerie, and D. Meyer. 1984. Inhibition of von Willebrand factor-platelet interaction by fibrinogen. Nature (Lond.). 308:648-649.

13. Gralnick, H. R., S. B. Williams, and B. S. Coller. 1984. Fibrinogen competes with von Willebrand factor for binding to the glycoprotein IIb/IIIa complex when platelets are stimulated with thrombin. Blood. 64:797-800.

14. Plow, E. F., A. H. Srouji, D. Meyer, G. Marguerie, and M. H. Ginsberg. 1984. Evidence that three adhesive proteins interact with a common recognition site on activated platelets. J. Biol. Chem. 259:53885391.

15. Plow, E. F., R. P. McEver, B. S. Coller, V. L. Woods, Jr., G. A. Marguerie, and M. H. Ginsberg. 1985. Related binding mechanisms for fibrinogen, fibronectin, von Willebrand factor, and thrombospondin on thrombin-stimulated human platelets. Blood. 66:724-727.

16. Liu, F-T., J. W. Bohn, E. L. Terry, H. Yamamoto, C. A. Molinaro, L. A. Sherman, N. R. Klinman, and D. H. Katz. 1980. Monoclonal anti-dinitrophenyl-specific murine IgE antibody: preparation, isolation and characterization. J. Immunol. 124:2728-2737.

17. Clamp, J. R., K. W. Hughes, and J. C. McPherson. 1971. Enzymelinked immunosorbent assay (ELISA) quantitative assay of immunoglobulin G. Immunochemistry. 8:871-879.

18. Coller, H. A., and B. S. Coller. 1983. Statistical analysis of repetitive subcloning by the limiting dilution technique with a view toward ensuring hybridoma monoclonality. Hybridoma. 2:91-96.

19. Montgomery, R. R., T. J. Kunicki, C. Taves, D. Pidard, and M. Corcoran. 1983. Diagnosis of Bernard-Soulier syndrome and Glanzmann's thrombasthenia with a monoclonal assay on whole blood. $J$. Clin. Invest. 71:385-389.

20. Ey, P. L., S. J. Prowse, and C. R. Jenkin. 1978. Isolation of pure IgG1, IgG2a and IgG2b immunoglobulins from mouse serum using protein A-Sepharose. Immunochemistry. 15:429-436.

21. Bruck, C., D. Portetelle, C. Glineur, and A. Bollen. 1982. Onestep purification of mouse monoclonal antibodies from ascitic fluid by DEAE Affi-Gel blue chromatography. J. Immunol. Methods. 53:313319.

22. Ouchterlony, O. 1964. Gel diffusion techniques. In Immunological Methods. J. F. Ackroyed, editor. F. A. Davis, Philadelphia. 55.

23. Nisonoff, A., and D. J. Dixon. 1964. Evidence for linkage of univalent fragments or half-molecules of rabbit $\gamma$-globulin by the same disulfide bond. Biochemistry. 3:1338-1342.

24. Parham, P. 1983. On the fragmentation of monoclonal IgG1, IgG2a, and IgG2b from BALB/c mice. J. Immunol. 131:2895-2902.

25. Porter, R. R. 1959. The hydrolysis of rabbit $\gamma$-globulin and antibodies with crystalline papain. Biochem. J. 73:119-126.

26. Laemmli, U. K. 1970. Cleavage of structural proteins during the assembly of the head of bacteriophage T4. Nature (Lond.). 227:680-685.

27. Lowry, O. H., N. J. Rosebrough, A. L. Farr, and R. J. Randall. 1951. Protein measurement with the folin phenol reagent. J. Biol. Chem. 193:265-275.

28. Walsh, P. N., D. C. B. Mills, and J. G. White. 1977. Metabolism and function of human platelets washed by albumin density gradient separation. Br. J. Haematol. 36:281-296.

29. Marguerie, G. A., T. E. Edgington, and E. F. Plow. 1980. Interaction of fibrinogen with its platelet receptor as part of a multistep reaction in ADP-induced aggregation. J. Biol. Chem. 255:154-161.

30. Tamura, G. S., M. O. Dailey, W. M. Gallatin, M. S. McGrath, I. L. Weissman, and E. A. Pillemer. 1984. Isolation of molecules rec- 
ognized by monoclonal antibodies and antisera: the solid phase immunoisolation technique. Anal. Biochem. 136:458-464.

31. Kunicki, T. J., D. Pidard, J-P. Rosa, and A. T. Nurden. 1981. The formation of $\mathrm{Ca}++$-dependent complexes of platelet membrane glycoproteins IIb and IIIa in solution as determined by crossed immunoelectrophoresis. Blood. 58:268-278.

32. Masri, M. A., S. A. Masri, and N. D. Boyd. 1983. Isolation of human fibrinogen of high purity and in high yield using polyethylene glycol 1000. Thromb. Haemostas. 49:116-119.

33. McKee, P. A., L. A. Rogers, E. Marler, and R. L. Hill. 1966. The subunit polypeptides of human fibrinogen. Arch. Biochem. Biophys. 116: 271-279.

34. Burnette, W. N. 1981. "Western Blotting": Electrophoretic transfer of proteins from sodium dodecyl sulfate-polyacrylamide gels to unmodified nitrocellulose and radiographic detection with antibody and radioiodinated protein A. Anal. Biochem. 112:195-203.

35. Newman, J., A. J. Johnson, M. H. Karpatkin, and S. Puszkin. 1971. Methods for the production of clinically effective intermediateand high-purity factor VIII concentrates. Br. J. Haematol. 21:1-20.

36. Switzer, M. E., and P. A. McKee. 1976. Studies on human antihemophilic factor. Evidence for a covalently linked subunit structure. J. Clin. Invest. 57:925-937.

37. Fraker, D. J., and J. C. Speck. 1978. Protein and cell membrane iodinations with a sparingly soluble chloroamide,1,3,4,6-tetrachloro3a,6a-diphenylglycoluril. Biochem. Biophys. Res. Commun. 80:849-857.

38. Scatchard, G. 1949. The attraction of proteins for small molecules and ions. Ann. NY Acad. Sci. 51:660-672.

39. Brass, L. F., and S. J. Shattil. 1982. Changes in surface-bound and exchangeable calcium during platelet activation. J. Biol. Chem. 257: 14000-14005.

40. Pidard, D., R. R. Montgomery, J. S. Bennett, and T. J. Kunicki. 1983. Interaction of AP-2, a monoclonal antibody specific for the human platelet glycoprotein Ilb-Illa complex, with intact platelets. J. Biol. Chem. 258:12582-12586.

41. Bennett, J. S., J. A. Hoxie, S. F. Leitman, G. Vilaire, and D. B. Cines. 1983. Inhibition of fibrinogen binding to stimulated human platelets by a monoclonal antibody. Proc. Natl. Acad. Sci. USA. 80:24172421.

42. Coller, B. S., E. I. Peerschke, L. E. Scudder, and C. A. Sullivan. 1983. A murine monoclonal antibody that completely blocks the binding of fibrinogen to platelets produces a thrombasthenic-like state in normal platelets and binds to glycoproteins IIb and/or IIIa. J. Clin. Invest. 72: 325-338.
43. Di Minno, G., P. Thiagarajan, B. Perussia, J. Martinez, S. S. Shapiro, G. Trinchieri, and S. Murphy. 1983. Exposure of platelet fibrinogen-binding sites by collagen, arachidonic acid, and ADP: Inhibition by a monoclonal antibody to the glycoprotein IIb-IIIa complex. Blood. 61:140-148.

44. McEver, R. P., E. M. Bennett, and M. N. Martin. 1983. Identification of two structurally and functionally distinct sites on human platelet membrane glycoprotein IIb-IIIa using monoclonal antibodies. J. Biol. Chem. 258:5269-5275.

45. Reynolds, J. A. 1979. Interaction of divalent antibody with cell surface antigens. Biochemistry. 18:264-269.

46. Mason, D. W., and A. F. Williams. 1980. The kinetics of antibody binding to membrane antigens in solution and at the cell surface. Biochem. J. 187:1-20.

47. Wencel-Drake, J. D., T. Kunicki, V. Woods, E. F. Plow, and M. H. Ginsberg. 1984. Intracellular pools of platelet membrane glycoproteins GPIb and GPIIb/III. Fed. Proc. 43:2193.

48. Brass, L. F., S. J. Shattil, T. J. Kunicki, and J. S. Bennett. 1985. Effect of calcium on the stability of the platelet membrane glycoprotein IIb-IIIa complex. J. Biol. Chem. 260:7875-7881.

49. Hagen, I., O. Bjerrum, G. Gogstad, R. Korsmo, and N. O. Solum. 1982. Involvement of divalent cations in the complex between the platelet glycoproteins Ilb and IIIa. Biochim. Biophys. Acta. 701:1-6.

50. Howard, L., S. Shulman, S. Sadanandan, and S. Karpatkin. 1982. Crossed immunoelectrophoresis of human platelet membranes. The major antigen consists of a complex of glycoproteins, GPIIb and GPIIIa, held together by $\mathrm{Ca}^{2+}$ and missing in Glanzmann's thrombasthenia. $J$. Biol. Chem. 257:8331-8336.

51. Jennings, L. K., and D. R. Phillips. 1982. Purification of glycoproteins IIb and III from human platelet plasma membranes and characterization of a calcium-dependent glycoprotein IIb-III complex. J. Biol. Chem. 257:10458-10466.

52. Zucker, M. B., D. Varon, N. C. Masiello, and S. Karpatkin. 1983. The combining ability of glycoproteins IIb, IIIa and $\mathrm{Ca}^{2+}$ in EDTAtreated nonaggregable platelets. Thromb. Haemostasis. 50:848-851.

53. Coller, B. S. 1985. A new murine monoclonal antibody reports an activation-dependent change in the conformation and/or microenvironment of the platelet glycoprotein IIb/IIla complex. J. Clin. Invest. 76:101-108.

54. Kloczewiak, M. A., S. D. Timmons, T. J. Lukas, and J. J. Hawiger. 1984. Structural attributes of synthetic peptides inhibiting and promoting aggregation of human platelets. Circulation. 70:II-358. 